\title{
Malaysia solar energy experience: intelligent fault location algorithm for unbalanced radial distribution network including PV systems
}

\begin{abstract}
Due to environmental issues and the upward trend of fossil fuel prices, the study of renewable energy (RE) based generation and their effects on the electrical system has become an important part of the government's energy policies and university projects. In RE generation, as solar photovoltaic (PV) systems are modular, silent, and transportable and demonstrate ease of installation, they have attracted a greater amount of attention specifically in those areas which receive considerable average solar radiation per day such as Malaysia. However, connecting solar PV farms to the grid like any other distributed generation (DG) units poses serious issues which arise in the distribution network. This paper presents a novel fault location algorithm based on the recording of short circuit power values at the primary substation of unbalanced radial distribution networks including PV systems. The recorded values are evaluated by a designed and tuned multi-layer feed forward neural network and the fault distances from the source are estimated accordingly. In order to highlight the accuracy of the presented method, the scenario is also repeated by recording the peak values of short circuit current which have been mostly used in the published intelligent fault location studies and the obtained results via two different values are compared with each other. The results reveal that the presented algorithm using a small scale input set is able to precisely locate different fault types in the unbalanced distribution networks including DG units.
\end{abstract}

Keyword: Malaysia solar energy; Intelligent fault location algorithm; Unbalanced radial distribution network; PV systems 